International Journal of Applied Mathematics

Volume 33 No. $3 \quad 2020,479-491$

ISSN: $1311-1728$ (printed version); ISSN: 1314-8060 (on-line version)

doi: http://dx.doi.org/10.12732/ijam.v33i3.8

\title{
UPPER AND LOWER SOLUTIONS METHOD FOR FRACTIONAL DIFFERENTIAL EQUATIONS WITH INTEGRAL BOUNDARY CONDITIONS
}

\author{
Jayshree Patil ${ }^{1}$, Archana Chaudhari ${ }^{2, *}$, Mohammed S. Abdo ${ }^{3}$, \\ Amol Bachhav ${ }^{1}$, Basel Hardan ${ }^{2}$ \\ ${ }^{1}$ Department of Mathematics, Vasantrao Naik Mahavidyalaya \\ Cidco, Aurangabad (M.S.), INDIA \\ 2 Department of Mathematics \\ Dr. Babasaheb Ambedkar Marathwada University \\ Aurangabad 431004 (M.S.), INDIA \\ ${ }^{3}$ Department of Mathematics, Hodeidah University \\ Al-Hodeidah, YEMEN
}

Abstract: In this paper, we investigate the existence and uniqueness of positive solutions of boundary value problems (BVPs) for fractional differential equations (FDEs) with boundary conditions (BCs) involving the RiemannLiouville (RL) fractional derivative of the form:

$$
\left\{\begin{array}{c}
-D_{0+}^{\sigma} x(t)=f(t, x(t)), \quad 0<t<1, \\
x(0)=x^{\prime}(0)=\ldots=x^{(n-2)}(0)=0, \\
x(1)=\vartheta \int_{0}^{1} x(s) d s, \quad 0<\vartheta<\sigma,
\end{array}\right.
$$

where $2 \leq n-1<\sigma \leq n$ and $\sigma \in \mathbb{R}$. The technique employed is coupled lower and upper solutions with fixed point theory on cone. An example is presented to justify our results.

AMS Subject Classification: 34K37; 26A33; 34A12; 47H10

Key Words: fractional differential equations; integral boundary condition; existence and uniqueness; upper and lower solutions; fixed point theorem

Received: February 20, 2020

(c) 2020 Academic Publications

${ }^{\S}$ Correspondence author 


\section{Introduction}

FDEs with BCs have applications in several fields such as applied mathematics, physics and engineering, see $[11,13,14,17]$. Existence theorems of multipoint have extensively studied using Leray-Schauder, coincidence degree theory, fixed point index theory, and fixed point theorems in cones. For more details, we refer the reader to $[10,12,19,22]$ and the references therein. On the other hand, investigating the existence and uniqueness of solutions and positive solutions of BVPs for nonlinear FDEs of the following type: functional, evolution, impulsive with integral BCs, can be found in $[3,4,2,1,5,6,7,8,9,15]$. For instance, Benchohra et al., in [7] studied some results on FDEs with mixed BCs of the form

$$
\left\{\begin{array}{l}
D_{0+}^{\sigma} x(t)=f(t, x(t)), \quad t \in I=[0, T], \\
x(0)+\mu \int_{0}^{T} x(s) d s=x(T),
\end{array}\right.
$$

where $0<\sigma \leq 1, D_{0+}^{\sigma}$ is the Caputo fractional derivative of order $\sigma, \mu \in \mathbb{R}$, and $f: I \times E \rightarrow E$ is a given function satisfying some conditions, $E$ is a Banach space with sup norm $\|\cdot\|$.

In [19] Wang et al., discussed the following nonlinear FDE with integral BCs

$$
\left\{\begin{array}{l}
D_{0+}^{\sigma} x(t)+f(t, x(t))=0, \quad t \in(0,1) \\
x(0)=0, \quad x(1)=\int_{0}^{1} x(s) d s
\end{array}\right.
$$

where $1<\sigma \leq 2, f:[0,1] \times[0, \infty) \rightarrow[0, \infty)$ is a continuous and $D_{0+}^{\sigma}$ is the standard RL fractional derivative.

Pawar and Salunke in [16] studied the existence and uniqueness of positive solutions of the following problem

$$
\left\{\begin{array}{c}
D_{0+}^{\sigma} x(t)=f(t, x(t)), \quad t \in I=[0,1], \\
x(0)+\vartheta \int_{0}^{1} x(s) d s=x(1),
\end{array}\right.
$$

where $D_{0+}^{\sigma}$ is the standard RL fractional derivative and $f:[0,1] \times[0,+\infty) \rightarrow$ $[0,+\infty)$ is a continuous.

Motivated by the above mentioned works, in this paper, we study the existence and uniqueness of solution for a class of FDE with BCs of the type

$$
\left\{\begin{array}{c}
-D_{0+}^{\sigma} x(t)=f(t, x(t)), \quad 0<t<1, \\
x(0)=x^{\prime}(0)=\ldots=x^{(n-2)}(0)=0, \\
x(1)=\vartheta \int_{0}^{1} x(s) d s, \quad 0<\vartheta<\sigma,
\end{array}\right.
$$

where $2 \leq n-1<\sigma \leq n$ is the order of RL fractional derivative $D_{0+}^{\sigma}$ and $0<\vartheta<\sigma$. 
To our knowledge, fewer works appeared to investigate in positive solutions to FDEs with BCs. Our analysis relies on the method of lower and upper solutions and some fixed point theorems on a cone. The results obtained can be more general, and some of them in the literature are as special cases, so they are recent contributions to this emerging field.

The rest of this paper is organized as follows: Section 2, devoted to the derivation of Green's function and its properties. Also, a discussion of preliminary facts is presented in this section. Section 3, deals with proof of existence result by using the lower and upper solutions for FDEs with BCs. The last section gives an example to demonstrate the main results.

\section{Preliminary results}

Definition 1. [13] For given a function $f:(0, \infty) \rightarrow \mathbb{R}$, then RL fractional integral of order $\sigma>0$ is given by

$$
I_{0+}^{\sigma} f(t)=\frac{1}{\Gamma(\sigma)} \int_{0}^{t}(t-s)^{\sigma-1} f(s) d s,
$$

provided that, the integral exists.

Definition 2. [13] For given a function $f:(0, \infty) \rightarrow \mathbb{R}$, then RL fractional derivative of order $\sigma>0$ is defined by

$$
D_{0+}^{\sigma} f(t)=\frac{1}{\Gamma(n-\sigma)}\left(\frac{d}{d t}\right)^{n} \int_{0}^{t}(t-s)^{n-\sigma-1} f(s) d s
$$

where $n-1<\sigma \leq n$, and the right side is pointwise defined on $(0, \infty)$ and $n=[\sigma]+1$.

Lemma 1. [11] Let $\sigma>0$ and $u \in C(0,1) \cap L(0,1)$. Then the linear FDE

$$
D_{0+}^{\sigma} u(t)=0
$$

has unique solution

$$
u(t)=c_{1} t^{\sigma-1}+c_{2} t^{\sigma-2}+\cdots+c_{n} t^{\sigma-n} .
$$

Moreover, if $u(t), D_{0+}^{\sigma} f(t) \in C(0,1) \cap L(0,1)$, then $I_{0+}^{\sigma} D_{0+}^{\sigma} u(t)=u(t)+c_{1} t^{\sigma-1}+$ $c_{2} t^{\sigma-2}+\cdots+c_{n} t^{\sigma-n}$, for some $c_{i} \in \mathbb{R}, i=1,2, \ldots, n$. 
Theorem 3. Let $n-1<\sigma<n$ and $\vartheta \neq \sigma$. Assume $u(t) \in C[0,1]$, then the unique solution of following problem

$$
\begin{gathered}
D_{0+}^{\sigma} x(t)=u(t), \quad 0<t<1, \\
x(0)=x^{\prime}(0)=\ldots=x^{(n-2)}(0)=0, \quad x(1)=\vartheta \int_{0}^{1} x(s) d s,
\end{gathered}
$$

is given by

$$
x(t)=\int_{0}^{1} G(t, s) u(s) d s,
$$

where $G(t, s)$ is the Green's function defined by

$$
G(t, s)=\left\{\begin{array}{l}
\frac{[t(1-s)]^{\sigma-1}(\sigma-\vartheta+\vartheta s)-(\sigma-\vartheta)(t-s)^{\sigma-1}}{(\sigma-\vartheta) \Gamma(\sigma)}, 0 \leq s \leq t \leq 1, \\
\frac{[t(1-s)]^{\sigma-1}(\sigma-\vartheta+\vartheta s)}{(\sigma-\vartheta) \Gamma(\sigma)}, 0 \leq t \leq s \leq 1
\end{array}\right.
$$

Proof. Applying $I_{0^{+}}^{\sigma}$ on both sides of (2). It follows from lemma 1 that

$$
x(t)=-\int_{0}^{t} \frac{(t-s)^{\sigma-1}}{\Gamma(\sigma)} u(s) d s+c_{1} t^{\sigma-1}+c_{2} t^{\sigma-2}+\cdots+c_{n} t^{\sigma-n},
$$

where $c_{1}, c_{2}, \ldots, c_{n} \in \mathbb{R}$. From (3), we have $c_{2}=c_{3}=\ldots=c_{n}=0$. Then the general solution of (2) is

$$
x(t)=-\int_{0}^{t} \frac{(t-s)^{\sigma-1}}{\Gamma(\sigma)} u(s) d s+c_{1} t^{\sigma-1} .
$$

Hence, by the condition $x(1)=\vartheta \int_{0}^{1} x(s) d s$, we get

$$
c_{1}=\int_{0}^{1} \frac{(1-s)^{\sigma-1}}{\Gamma(\sigma)} u(s) d s+\vartheta \int_{0}^{1} x(s) d s
$$

Put the value of $c_{1}$ in (5), we obtain

$$
\begin{aligned}
x(t)= & -\int_{0}^{t} \frac{(t-s)^{\sigma-1}}{\Gamma(\sigma)} u(s) d s+t^{\sigma-1} \int_{0}^{1} \frac{(1-s)^{\sigma-1}}{\Gamma(\sigma)} u(s) d s \\
& +\vartheta t^{\sigma-1} \int_{0}^{1} x(s) d s .
\end{aligned}
$$


Let $\mathcal{A}:=-\int_{0}^{1} x(s) d s$. By the formula (6) and Fubini's theorem, we get

$$
\begin{aligned}
\int_{0}^{1} x(t) d t= & -\int_{0}^{1} \int_{0}^{t} \frac{(t-s)^{\sigma-1}}{\Gamma(\sigma)} u(s) d s d t \\
& +\int_{0}^{1} t^{\sigma-1} \int_{0}^{1} \frac{(1-s)^{\sigma-1}}{\Gamma(\sigma)} u(s) d s d t+\vartheta \mathcal{A} \int_{0}^{1} t^{\sigma-1} d t \\
= & \int_{0}^{1} \frac{s(1-s)^{\sigma-1}}{\sigma \Gamma(\sigma)} u(s) d s+\frac{\vartheta \mathcal{A}}{\sigma}
\end{aligned}
$$

which implies

$$
\mathcal{A}=\int_{0}^{1} \frac{s(1-s)^{\sigma-1}}{(\sigma-\vartheta) \Gamma(\sigma)} u(s) d s .
$$

Substitute value of $\mathcal{A}$ from (7) into (6), we have

$$
\begin{aligned}
& x(t) \\
= & \int_{0}^{1}\left[\frac{\sigma-\vartheta+\vartheta s}{\sigma-\vartheta}\right] \frac{[(1-s) t]^{\sigma-1}}{\Gamma(\sigma)} u(s) d s-\int_{0}^{t} \frac{(t-s)^{\sigma-1}}{\Gamma(\sigma)} u(s) d s \\
= & \int_{0}^{t}\left[\frac{\sigma-\vartheta+\vartheta s}{\sigma-\vartheta}\right] \frac{[(1-s) t]^{\sigma-1}}{\Gamma(\sigma)} u(s) d s-\int_{0}^{t} \frac{(t-s)^{\sigma-1}}{\Gamma(\sigma)} u(s) d s \\
& +\int_{t}^{1}\left[\frac{\sigma-\vartheta+\vartheta s}{\sigma-\vartheta}\right] \frac{[(1-s) t]^{\sigma-1}}{\Gamma(\sigma)} u(s) d s \\
= & \int_{0}^{t} \frac{\left[(\sigma-\vartheta+\vartheta s)[(1-s) t]^{\sigma-1}-(\sigma-\vartheta)(t-s)^{\sigma-1}\right]}{(\sigma-\vartheta) \Gamma(\sigma)} u(s) d s \\
& +\int_{t}^{1} \frac{(\sigma-\vartheta+\vartheta s)[(1-s) t]^{\sigma-1}}{(\sigma-\vartheta) \Gamma(\sigma)} u(s) d s \\
= & \int_{0}^{1} G(t, s) u(s) d s .
\end{aligned}
$$

Lemma 2. For all $\sigma \in(n-1, n]$ and $\vartheta \geq 0$. The Green function given by (4) satisfies the following properties:

(i) $G(t, s) \geq 0, \quad t, s \in(0,1)$.

(ii) $(\sigma-\vartheta) G(1, s)>0$, for all $s \in(0,1)$ if and only if $\sigma \neq \vartheta$.

(iii) $G(t, s) \leq \frac{1}{(\sigma-\vartheta) \Gamma(\sigma)}$ for all $t, s \in[0,1]$ and $\vartheta \in[0, \sigma)$.

(iv) For $n-1<\sigma \leq n$ and $0<\vartheta<\sigma$, we have

$$
t^{\sigma-1} G(1, s) \leq G(t, s) \leq \frac{\sigma}{\vartheta} G(1, s), \quad t, s \in(0,1) .
$$


Proof. The proofs of (i), (ii) and (iii) are easy, so we omit it. In the following, we shall prove (iv) in two cases :

Case (i). In $0<t<s<1$,

$$
\frac{G(t, s)}{G(1, s)}=t^{\sigma-1} \leq t^{\sigma-1}\left(1+\frac{\sigma-\vartheta}{\vartheta s}\right) \leq t^{\sigma-1} \frac{\sigma}{\vartheta s} \leq t^{\sigma-2} \frac{\sigma}{\vartheta}<\frac{\sigma}{\vartheta}
$$

Case (ii). In $0<s<t<1$,

$$
\frac{G(t, s)}{G(1, s)}=\frac{t^{\sigma-1}(1-s)^{\sigma-1}(\sigma-\vartheta+\vartheta s)-(\sigma-\vartheta)(t-s)^{\sigma-1}}{(1-s)^{\sigma-1}(\sigma-\vartheta+\vartheta s)-(\sigma-\vartheta)(1-s)^{\sigma-1}} .
$$

Since $s \geq t s$, we deduce that

$$
\begin{aligned}
\frac{G(t, s)}{G(1, s)} & \geq \frac{t^{\sigma-1}\left[(1-s)^{\sigma-1}(\sigma-\vartheta+\vartheta s)-(\sigma-\vartheta)(1-s)^{\sigma-1}\right]}{(1-s)^{\sigma-1}(\sigma-\vartheta+\vartheta s)-(\sigma-\vartheta)(1-s)^{\sigma-1}} \\
& =t^{\sigma-1} .
\end{aligned}
$$

Now from (ii) the inequality (8) is satisfied.

Lemma 3. Let $D$ be a subset of the cone $K$ of semi-order Banach space $E, T: D \rightarrow E$ be nondecreasing. If there exists $x_{0}, y_{0} \in D$ such that $x_{0} \leq y_{0}$, $\left(x_{0}, y_{0}\right) \subset D$ and $x_{0}, y_{0}$ are the lower and upper solutions of equation $x-T(x)=$ 0 , then the equation $x-T(x)=0$ has maximum solution and minimum solution $x^{*}, y^{*}$ in $\left(x_{0}, y_{0}\right)$ such that $x^{*} \leq y^{*}$, when one of the following assumptions hold:

(i) $T$ is compact continuous and $K$ is normal;

(ii) $T$ is continuous and $K$ is regular;

(iii) $T$ is continuous or weak continuous and $E$ is reflexive, $K$ is normal.

Now, let $E=C[0,1]$. Then $E$ is a Banach space endowed with norm $\|x\|=$ $\sup _{t \in[0,1]}|x(t)|$. The Cone $K \subset E$ is defined by

$$
K=\{x \in E \mid x(t) \geq 0,0 \leq t \leq 1\} .
$$

Assume that $u(t)=f(t, x(t))$, then it follows from Theorem 3 that the problem (1) has a solution if and only if the operator $T$ defined by

$$
T(x)=\int_{0}^{1} G(t, s) f(s, x(s)) d s
$$

has a fixed point. First of all, we give the definition of lower and upper solutions of the operator $T$. 
Definition 4. The function $v(t) \in E$ is called a lower solution of operator $T$ if

$$
D^{\sigma} v(t) \leq f(t, v(t)), v(t) \leq T v(t), 0<t<1,
$$

and the function $w(t) \in E$ is called a upper solution of operator $T$ if

$$
D^{\sigma} w(t) \geq f(t, w(t)), w(t) \geq T w(t), 0<t<1 .
$$

We consider the following set of assumptions:

$\left(A_{1}\right) f:[0,1] \times[0,+\infty) \rightarrow[0,+\infty)$ is continuous, $f(t, \cdot)$ is nondecreasing for each $t \in[0,1]$, and there exists a positive constant $k$ such that $f(t, \cdot)$ is strictly increasing on $[0, k]$ for each $t \in[0,1]$.

$\left(A_{2}\right) \quad 0<\lim _{x \rightarrow+\infty} f(t, x(t))<+\infty$, for each $t \in[0,1]$.

$\left(A_{3}\right) v_{0}$ and $w_{0}$ are a lower and upper solutions of problem (1) satisfying $v_{0} \leq$ $w_{0}, 0 \leq t \leq 1$.

\section{Main results}

In this section, we prove the existence and uniqueness of positive solution of the problem (1).

Theorem 5. (Existence Theorem) Assume that $\left(A_{1}\right)-\left(A_{3}\right)$ hold. Then the problem (1) has a positive solution.

Proof. We will give proof in the following four steps

Step 1. $T: K \rightarrow K$ is completely continuous.

At first, $T: K \rightarrow K$ is continuous according to continuity and non-negativeness of $G(t, s)$ and $f(t, x)$. Next, Let $M \subset K$ be a bounded set, i.e., there exists a positive constant $\ell>0$ such that $\|x\| \leq \ell ; \forall x \in M$. Let $L=\max _{0 \leq t \leq 1,0 \leq x \leq \ell}|f(t, x)|+$ 1. Then, for every $x \in M$, we get

$$
|T x(t)| \leq \int_{0}^{1} G(t, s)|f(s, x(s))| d s \leq \frac{L}{(\sigma-\vartheta) \Gamma(\sigma)}, \forall t \in[0,1] .
$$


Consequently, $T(M)$ is bounded. Finally, we set $x \in M$, then for each $t \in[0,1]$ the following inequalities are satisfied by means of Lemma 2 :

$$
\begin{aligned}
T x(t) & =\int_{0}^{1} G(t, s) f(s, x(s)) d s \geq t^{\sigma-1} \int_{0}^{1} G(1, s) f(s, x(s)) d s \\
& \geq \frac{t^{\sigma-1} \vartheta}{\sigma} \int_{0}^{1} \max _{t \in[0,1]}\{G(t, s)\} f(s, x(s)) d s \\
& \geq \frac{t^{\sigma-1} \vartheta}{\sigma} \max _{t \in[0,1]}\left\{\int_{0}^{1} G(t, s) f(s, x(s)) d s\right\}=\frac{t^{\sigma-1} \vartheta}{\sigma}\|T x\|,
\end{aligned}
$$

and

$$
\begin{aligned}
\left|(T x)^{\prime}(t)\right|= & \mid-\int_{0}^{t} \frac{(t-s)^{\sigma-2}}{\Gamma(\sigma-1)} f(s, x(s)) d s \\
& +\int_{0}^{1} \frac{(\sigma-\vartheta+\vartheta s)(1-s)^{\sigma-1} t^{\sigma-2}}{(\sigma-\vartheta) \Gamma(\sigma-1)} f(s, x(s)) d s \mid \\
\leq & \frac{L}{\Gamma(\sigma-1)} \int_{0}^{t}(t-s)^{\sigma-2} d s+\frac{L}{\Gamma(\sigma-1) \sigma} \\
& +\frac{\vartheta L}{(\sigma-\vartheta) \Gamma(\sigma-1)} \int_{0}^{1} s(1-s)^{\sigma-1} d s \\
\leq & \frac{L}{\Gamma(\sigma)}+\frac{L}{\Gamma(\sigma-1)}+\frac{L \vartheta}{(\sigma-\vartheta) \Gamma(\sigma-1) \sigma(\sigma+1)}:=N .
\end{aligned}
$$

Hence, for all $t_{1}, t_{2} \in[0,1]$ with $t_{1}<t_{2}$, we obtain

$$
\left\|T x\left(t_{2}\right)-T x\left(t_{1}\right)\right\| \leq \int_{t_{1}}^{t_{2}}\left|(T x)^{\prime}(s)\right| d s \leq N\left(t_{2}-t_{1}\right) .
$$

Thus, $T(M)$ is an equicontinuous. As a consequence of Arzela-Ascoli Theorem, we conclude that $\overline{T(M)}$ is compact. Therefore $T: K \rightarrow K$ is completely continuous.

Step 2. The operator $T$ is an increasing.

Let $x_{0}, y_{0} \in K$ with $x_{0} \leq y_{0}$. Then by $\left(A_{1}\right)$, we obtain

$$
T x_{0}(t)=\int_{0}^{1} G(t, s) f\left(s, x_{0}(s)\right) d s \leq \int_{0}^{1} G(t, s) f\left(s, y_{0}(s)\right) d s \leq T y_{0}(t) .
$$

Thus, $T$ is an increasing operator. Operation the definition of the lower and upper solution, we obtain $T x_{0} \geq x_{0}$ and $T y_{0} \leq y_{0}$. Hence $T:\left(x_{0}, y_{0}\right) \rightarrow\left(x_{0}, y_{0}\right)$ is a compact continuous operator. 
Step 3. By $\left(A_{2}\right)$, there exists positive constants $M_{1}$ and $N$ such that $x \geq N$ it holds $f(t, x(t)) \leq M_{1}$.

But in exchange, since $f:[0,1] \times[0, N]$ is continuous (by $\left(A_{1}\right)$ ), there exists a positive constant $M_{2}>0$ such that $f(t, x(t)) \leq M_{2}$. Let $M=\max \left\{M_{1}, M_{2}\right\}$. Then we have $f(t, x(t)) \leq M, \forall x \geq 0$.

Now, let us take the following problem

$$
\left\{\begin{array}{l}
D_{0+}^{\sigma} w(t)+M=0,0<t<1,2 \leq n-1<\sigma \leq n \\
w(0)=w^{\prime}(0)=\ldots=w^{(n-2)}(0)=0, w(1)=\vartheta \int_{0}^{1} w(s) d s
\end{array}\right.
$$

where $0<\vartheta<\sigma$ and $\sigma \in \mathbb{R}$. From the Theorem 3, we have the solution of (9) is

$$
w(t)=\int_{0}^{1} G(t, s) M d s \geq \int_{0}^{1} G(t, s) f(s, w(s)) d s=T w(t),
$$

which means that $w(t)$ is an upper solution of $T$.

On the other hand, it is obvious that $v(t) \equiv 0$ is a lower solution of $T$. Hence $v(t) \leq w(t)$.

Step 4. Since $K$ is a normal cone, and by utilize Lemma 3, we deduce that there exists a fixed point $x(t) \in(0, w(t))$ of $T$. Therefore, the problem (1) has a positive solution, which completes the proof.

Theorem 6. (Uniqueness Theorem) Assume that $f$ satisfies

$$
|f(t, x)-f(t, y)| \leq b(t)|x-y|, \forall t \in[0,1], x, y \in \mathbb{R}^{+},
$$

where $b:[0,1] \rightarrow \mathbb{R}^{+}$is a continuous. If

$$
\int_{0}^{1} s^{\sigma-1}(1-s)^{\sigma-1}(\sigma-\vartheta+\vartheta s) b(s) d s<(\sigma-\vartheta) \Gamma(\sigma),
$$

then the problem (1) has unique positive solution.

Proof. We known that the problem (1) has a unique positive solution if the operator $T^{m}$ is a contraction mapping for $m$ sufficiently large. Indeed, by the definition of Green's function $G(t, s)$, then for $x, y \in K$, we have the following estimate

$$
\begin{aligned}
& |T x(t)-T y(t)| \\
= & \left|\int_{0}^{1} G(t, s) f(s, x(s)) d s-\left(\int_{0}^{1} G(t, s) f(s, y(s)) d s\right)\right|
\end{aligned}
$$




$$
\begin{aligned}
& \leq \int_{0}^{1} G(t, s)|f(s, x(s))-f(s, y(s))| d s \\
& \leq \int_{0}^{1} G(t, s) b(s)|x(s)-y(s)| d s \\
& \leq \int_{0}^{1} \frac{\|x-y\|}{(\sigma-\vartheta) \Gamma(\sigma)}[t(1-s)]^{\sigma-1}(\sigma-\vartheta+\vartheta s) b(s) d s \\
& \leq \frac{\|x-y\| t^{\sigma-1}}{(\sigma-\vartheta) \Gamma(\sigma)} \int_{0}^{1}(1-s)^{\sigma-1}(\sigma-\vartheta+\vartheta s) b(s) d s .
\end{aligned}
$$

Let $I=\int_{0}^{1}(1-s)^{\sigma-1}(\sigma-\vartheta+\vartheta s) b(s) d s$. Then we have

$$
|T x(t)-T y(t)| \leq \frac{I t^{\sigma-1}}{(\sigma-\vartheta) \Gamma(\sigma)}\|x-y\| .
$$

Similarly,

$$
\begin{aligned}
& \left|T^{2} x(t)-T^{2} y(t)\right| \\
= & \int_{0}^{1} G(t, s)|f(s, T x(s))-f(s, T y(s))| d s \\
\leq & \int_{0}^{1} G(t, s) b(s)|T x(s)-T y(s)| d s \\
\leq & \int_{0}^{1} G(t, s) a(s) \frac{I t^{\sigma-1}}{(\sigma-\vartheta) \Gamma(\sigma)}\|x-y\| d s \\
\leq & \frac{I\|x-y\| t^{\sigma-1}}{(\sigma-\vartheta)^{2} \Gamma^{2}(\sigma)} \int_{0}^{1} s^{\sigma-1}(1-s)^{\sigma-1}(\sigma-\vartheta+\vartheta s) b(s) d s .
\end{aligned}
$$

Let $J=\int_{0}^{1} s^{\sigma-1}(1-s)^{\sigma-1-q} b(s) d s$. Then

$$
\left|T^{2} x(t)-T^{2} y(t)\right| \leq \frac{I J t^{\sigma-1}}{(\sigma-\vartheta)^{2} \Gamma^{2}(\sigma)}\|x-y\| .
$$

It follows from the mathematical induction that

$$
\left|T^{m} x(t)-T^{m} y(t)\right| \leq \frac{I J^{m-1} t^{\sigma-1}}{(\sigma-\vartheta)^{m} \Gamma^{m}(\sigma)}\|x-y\| .
$$

For sufficiently large $m$, by (10), we have

$$
\frac{I J^{m-1}}{(\sigma-\vartheta)^{m} \Gamma^{m}(\sigma)}=\frac{I}{(\sigma-\vartheta) \Gamma(\sigma)}\left(\frac{J}{(\sigma-\vartheta) \Gamma(\sigma)}\right)^{m-1}<1 .
$$


Hence, it holds

$$
\left\|T^{m} x(t)-T^{m} y(t)\right\|<\|x-y\|
$$

which implies the operator $T^{m}$ is a contraction mapping for $m$ sufficiently large. So, the problem (1) has a unique positive solution. This completes the proof.

Example 1. Consider the following nonlinear FDE with integral BCs

$$
\left\{\begin{array}{l}
-D_{0+}^{\frac{7}{2}} x(t)=\frac{1}{(t+4)^{2}}\left(\frac{|x(t)|}{1+|x(t)|}+5 t^{2}\right), 0<t<1 \\
x(1)=2 \int_{0}^{1} x(s) d s, x^{(k)}(0)=0, k=0,1,2
\end{array}\right.
$$

where $\sigma=\frac{7}{2}(n=4), \vartheta=2, f(t, x)=\frac{1}{(t+4)^{2}}\left(\frac{|x|}{1+|x|}+5 t^{2}\right)$. It is easy to see that $f$ is continuous and nonnegative function. Moreover, for $x, y \in \mathbb{R}^{+}, \mid f(t, x)-$ $f(t, y)\left|\leq \frac{1}{(t+4)^{2}}\right| x-y \mid$. Therefore, Theorem 6 is satisfied with $b(t)=\frac{1}{(t+4)^{2}} \in$ $C\left([0,1], \mathbb{R}^{+}\right)$. In addition, by some direct calculation, we find that

$$
\int_{0}^{1} s^{\sigma-1}(1-s)^{\sigma-1}(\sigma-\vartheta+\vartheta s) b(s) d s \approx 0.002<\frac{45 \sqrt{ } \pi}{16}=(\sigma-\vartheta) \Gamma(\sigma) .
$$

An application of Theorem 6 implies that the problem (11) has a unique positive solution. On the other hand, it is clear that $f(t, \cdot)=\frac{1}{(t+4)^{2}}\left(\frac{|\cdot|}{1+|\cdot|}+5 t^{2}\right)$ is nondecreasing for each $t \in[0,1]$. Also,

$$
0<\lim _{x \rightarrow \infty}\left(\frac{1}{(t+4)^{2}}\left(\frac{|x(t)|}{1+|x(t)|}+5 t^{2}\right)\right)<\frac{5}{16}<+\infty\left(M=\frac{5}{16}\right) .
$$

Finally,

$$
w_{0}(t)=\int_{0}^{1} G(t, s) M d s=\frac{225}{256} \sqrt{ } \pi\left(\frac{5 t^{\frac{5}{2}}}{9}-\frac{3}{7}\left(t^{\frac{7}{2}}-(t-1)^{\frac{7}{2}}\right)\right), 0<t<1
$$

is an upper solution of (11). Obviously, $v_{0}(t)=0$ is a lower solution of (11) and $v_{0}(t) \leq w_{0}(t), \quad 0 \leq t \leq 1$. Thus all the conditions $\left(A_{1}\right)-\left(A_{3}\right)$ are satisfied. Hence, by Theorem 5, the FDE (11) has a positive solution on [0,1]. 


\section{References}

[1] M.S. Abdo, S.K. Panchal, Caputo fractional integro-differential equation with nonlocal conditions in Banach space, Int. J. Appl. Math. (IJAM), 32, No 2 (2019), 279-288; DOI: 10.12732/ijam.v32i2.9.

[2] M.S. Abdo, S.K. Panchal, Existence and continuous dependence for fractional neutral functional differential equations, J. Mathematical Model., 5, No 2 (2017), 153-170.

[3] M.S. Abdo, S.K. Panchal, A.M. Saeed, Fractional boundary value problem with $\psi$-Caputo fractional derivative, Proceedings - Math. Sci., 129, No 5 (2019), 65 .

[4] M.S. Abdo, H.A. Wahash, S.K. Panchal, Positive solution of a fractional differential equation with integral boundary conditions, J. Appl. Math. Computational Mech., 17, No 3 (2018), 5-15.

[5] S. Adil, S.B. Hadid, Existence and uniqueness theorem for fractional differential equation with integral boundary condition, J. Fract. Calc. Appl., 3, No 6 (2012), 1-9.

[6] A. Ardjouni, A. Djoudi, Existence and uniqueness of positive solutions for first-order nonlinear Liouville-Caputo fractional differential equations, $S \tilde{a} o$ Paulo J. Math. Sci., 14 (2020), 381390. https://doi.org/10.1007/s40863019-00147-2

[7] M. Benchohra, F.Z. Mostefai, Weak solution for nonlinear fractional differential equation with integral boundary condition in Banach spaces, Elect. J. Qualit. Theory Diff. Equ., 2010, No 54 (2010), 1-10.

[8] A. Benmezai, A. Saadi, Existence of positive solutions for a nonlinear fractional differential equations with integral boundary conditions, J. Fract. Calc. Appl., 7, No 2 (2016), 145-152.

[9] H. Boulares, A. Ardjouni, Y. Laskri, Positive solutions for nonlinear fractional differential equations, Positivity, 21, No 3 (2017), 1201-1212.

[10] C. Cheng, Z. Feng, Y. Su, Positive solution of fractional differential equation with derivative terms, Elect. J. Diff. Equ., 215 (2012), 1-27.

[11] K. Diethelm, N.J. Ford, Analysis of fractional differential equations, J. Math. Anal. Appli., 265, No 2 (2002), 229-248. 
[12] X. Hao, L. Liu, Y. Wu, Positive solution of nonlinear fractional semipositone differential equation with nonlocal boundary condition, J. Nonlinear Sci. Appl., 9, No 6 (2016), 3992-4002.

[13] A.A. Kilbas, H.M. Srivastava, J.J. Trujillo, Theory and applications of fractional differential equations, Elsevier Science BV, Amersterdam, (2006).

[14] V. Lakshmikantham, S. Leele, J.V. Devi, Theory and applications of fractional dynamical systems, Cambridge Scientific Publishers Ltd, (2009).

[15] M.A. Malahi, M.S. Abdo, S.K. Panchal, Positive solution of Hilfer fractional differential equations with integral boundary conditions, arXiv:1910.0788\%, (2019), 1-10.

[16] G.U. Pawar, J.N. Salunke, Upper and lower solution method for nonlinear fractional differential equations boundary value problem, J. Comput. Math. Sci., 9, No 3 (2018), 164-173.

[17] I. Podlubny, Fractional differential equations, Academic Press, San Diego, (1999).

[18] K. Shah, R.A. Khan, Iterative solutions to a coupled system of nonlinear fractional differential equation, J. Frac. Calc. Appl., 7, No 2 (2016), 40-50.

[19] G. Wang, S. Liu, R.P. Agrawal, L. Zhang, Positive solution of integral boundary value problem involving Riemann-Liouville fractional derivative, J. Frac. Calc. Appl., 4, No 2 (2013), 312-321.

[20] T. Wang, F. Xie, Existence and uniqueness of fractional differential equation with integral boundary conditions, J. Nonlinear Sci. Appl., 1, No 4 (2008), 2006-2012.

[21] X. Wang, L. Wang, Q. Zeng, Fractional differential equations with integral boundary conditions, J. Nonlinear Sci. Appl., 8, No 4 (2015), 309-314.

[22] D. Zhao, Y. Liu, Positive solution for a class of fractional differential coupled system with integral boundary value condition, J. Nonlinear Sci. Appl., 9, No 5 (2016), 2922-2942. 
